\title{
Impact of hearing impairment on psychological distress and subjective well-being in older adults
}

\author{
Yasmeen Niazi ${ }^{1}$, Bisma Ejaz ${ }^{2}$, \\ Amina Muazzam ${ }^{3}$
}

\begin{abstract}
Objective: The main objective of this study was to explore the impact of hearing impairment on psychological distress and subjective well-being in older adults with hearing impairment.

Methods: The study with cross sectional research design was conducted in three public sector hospitals of Lahore, from February 2017 to June 2017. Participants of the study were adults aged 50-90 years and with hearing impairment, selected through non-probability sampling technique. Demographic Information sheet, Kessler psychological distress scale by Kessler, Mroczek. in 1992 and Satisfaction with life scale by Diener, Emmons, Larsen, Griffin in 1985 were used for data collection. SPSS 21 was used to analyze the data.

Results: There were 200 participants with age ranged from 53 to 89 years $(M=65.92, S D=9.70)$. Of the total, $100(50 \%)$ subjects were men and $100(50 \%)$ were women. Significant gender differences were found in psychological distress, with men reflecting more symptoms of psychological distress $(p<0.01)$, whereas non-significant gender differences were found in case of subjective well-being $(p>0.05)$. Moreover, psychological distress was observed as a predictor of subjective well-being $(p<0.01)$. One-way analysis of variance revealed insignificant differences of psychological distress and subjective well-being across three levels of hearing impairment.

Conclusion: Early diagnosis and rehabilitation of age-related hearing loss improves the overall quality of life of older adults living with hearing impairment.
\end{abstract}

KEYWORDS: Psychological distress, Subjective well-being, Hearing impairment.

How to cite this:

doi: https://doi.org/10.12669/pjms.36.6.2457

Niazi Y, Ejaz B, Muazzam A. Impact of hearing impairment on psychological distress and subjective well-being in older adults. Pak J Med Sci. 2020;36(6):1210-1215. doi: https://doi.org/10.12669/pjms.36.6.2457

This is an Open Access article distributed under the terms of the Creative Commons Attribution License (http://creativecommons.org/licenses/by/3.0), which permits unrestricted use, distribution, and reproduction in any medium, provided the original work is properly cited.

1. Yasmeen Niazi, MS Scholar.

2. Bisma Ejaz,

Assistant Professor,

3. Amina Muazzam

Tenured Associate Professor,

1-3: Department of Applied Psychology,

Lahore College for Women University,

Lahore, Pakistan.

Correspondence:

Dr. Amina Muazzam

Tenured Associate Professor

Department of Applied Psychology,

Lahore College for Women University,

Lahore, Pakistan.

Email: aminamuazzzam3@gmail.com

* Received for Publication:

March 3, 2020

* $1^{\text {st }}$ Revision Received:

* $2^{\text {nd }}$ Revision Received:

* Final Revision Accepted:

April 6, 2020

July 22, 2020

July 23,2020

\section{INTRODUCTION}

Hearing impairment is described as the partial or total loss of hearing ability. On the basis of the severity of the problem hearing impairment can be classified as mild, moderate, severe and profound. Age related hearing impairment is a very common disorder in older adulthood. It is considered as third most prevalent chronic health condition in people over 65 years of age. ${ }^{1}$ Hearing impairment in old age typically stems from some pathological changes inside the ear which are related to aging, it may lead to the impairment of low to high frequency hearing. This problem comes up with greater difficulty in listening process that significantly impairs person's ability 
to communicate with others, which in turn is a serious concern related to the mental health of individual. ${ }^{2}$ Hearing impairment in adults is specified by threshold greater than $40 \mathrm{dBHL}$ in better hearing ear. There is no proven treatment for the age related hearing loss, the only way to help people with hearing loss is the use of assistive techniques (hearing aids and cochlear implants) that can increase the threshold to a significant level but do not return hearing to normal. ${ }^{3}$

Hearing impairment in aging is substantially associated with disability, increased risk of incident morbidity, poor self-perceived health, ${ }^{4}$ poor psychological well-being, low levels of self-efficacy and happiness. ${ }^{5}$ The problem is also proved to be linked with anxiety, cognitive decline and lower health related quality of life. ${ }^{6}$ Aforementioned symptoms and their morbidity were found conceivably related to the severity of hearing impairment itself. ${ }^{7}$

Psychological distress is defined as the condition of emotional disturbance with feelings of anxiety (e.g., feeling restless and tense), depression (e. g., feeling hopeless and loss of interest). People may experience psychological distress while coping with the stressful, disturbing or harmful circumstances in their daily life. ${ }^{8}$ While studying the psychological distress in hearing impaired older adults it was reported that they were more vulnerable to mental health problems like depression, decreased wellbeing, emotional sensitivity and aggression. ${ }^{9}$ Hearing loss can prevent people from being exposed to socially challenging circumstances resulting in isolation which significantly impinges upon their quality of life and mental health. ${ }^{10}$ Perceived social disability has been proved to serve as a mediator between hearing impairment and psychological distress including aggression. ${ }^{11}$ It has been reported that adults with hearing loss tend to exhibit more symptoms of depression, anxiety, psychological distress, and emotional sensitivity as compared to people with normal hearing. ${ }^{12}$

Subjective well-being is how a person evaluates his/her life cognitively and emotionally as a whole, it basically includes cognitive judgments concerning life satisfaction and emotional reactions to life events that may be positive or negative. ${ }^{13}$ Hearing loss poses a serious risk to the mental wellbeing and overall quality of life in older adults. People may experience diminished self-esteem that in effect leads to the poor mental health and decline in psychological well-being. ${ }^{14}$ In a study intended to find out the impact of hearing loss on cognitive and emotional domains specifically, most of the people described that hearing impairment has a significantly negative connotation and it accounts for the poor well-being in general. ${ }^{15}$ It is evident from the literature that higher well-being was significantly linked to decreased mental distress and social isolation in adult population with hearing impairment. The study provides a clear evidence that rehabilitation techniques and proper intervention plan not only have remarkable effect on well-being of people with hearing impairment but it also reduces the psychological distress to a greater extent. ${ }^{16}$

Current study was aimed to gauge the impact of age related hearing impairment on psychological distress and subjective well-being in older adults. Gender differences in psychological distress and subjective well-being were studied. Additionally, predictive relationship between psychological distress and subjective well-being and difference in both variables were investigated.

\section{METHODS}

The cross sectional study was conducted in Lahore, from February 2017 to June 2017. The study sample comprised of 200 older adults 53 to 89 years old with hearing impairment, among the participants $100(50 \%)$ were men and $100(50 \%)$ were women. Non-probability purposive sampling technique was employed to select the sample. The clinical diagnosis of hearing impairment was carried by the physicians using audiometry. People with hearing impairment but less than 50 years of age were excluded from the study.

A brief demographic form along with Kessler Psychological Distress Scale (K10) by Kessler and Satisfaction with Life scale (SWLS) developed by Diener and his colleagues, was used for data collection. Kessler Psychological Distress Scale (K10), was used to measure the mental condition of the participants. Scale consisted of 10 items graded on likert scale of five points $(1=$ none of the time to $5=$ all of the time). $\mathrm{K} 10$ was proved to be a better predictor of non specific psychological distress, anxiety and depression and is widely used as a global measure of psychological distress. The scale has good reliability, ranges from 0.89 to $0.91 .^{17}$

Subjective well-being of the participants was assessed by the Satisfaction with Life Scale (SWLS). This is a short form 5 item scale, responses were rated on 7 point likert scale $(1=$ strongly agree to $7=$ strongly disagree). It is widely used scale 
Impact of hearing impairment on psychological distress

Table-I: Independent sample t-test comprising gender differences in

PD and SWB among adults with hearing impairment $(n=200)$.

\begin{tabular}{|c|c|c|c|c|c|c|c|c|}
\hline \multirow[t]{2}{*}{ Variables } & \multicolumn{2}{|c|}{ Male $(n=100)$} & \multicolumn{2}{|c|}{ Female $(n=100)$} & \multirow[b]{2}{*}{$d f$} & \multirow[b]{2}{*}{$t$} & \multirow[b]{2}{*}{$p$} & \multirow[b]{2}{*}{ Cohen's d } \\
\hline & $M$ & $S D$ & $M$ & $S D$ & & & & \\
\hline PD & 33.05 & 5.19 & 31.62 & 3.54 & 198 & 2.27 & $0.02^{*}$ & 0.32 \\
\hline SWB & 18.90 & 4.27 & 18.86 & 4.05 & 198 & 0.068 & 0.94 & 0.01 \\
\hline
\end{tabular}

$\mathrm{PD}=$ Psychological distress, $\mathrm{SWB}=$ Subjective well-being ${ }^{*} \mathrm{p}<0.05$.

for measuring life satisfaction having good psychometric properties. The reliability of scale was reported by authors as $0.87 .{ }^{18}$

Prior to data collection informed consent was obtained from the participants after elaborating the purpose of the study, they were assured about the confidentiality of their personal information. Urdu versions of Kessler Psychological Distress Scale (K10) and Satisfaction with Life Scale (SWLS) were administered to collect data about study variables. Personal demographics of patients were obtained on separate sheet prepared to collect information regarding age, gender, marital status, family system, socio-economic status and necessary details about their problem. SPSS 21 version was used for data analysis. Analysis of the basic variables was done by using descriptive statistics. Gender differences among two study variables were studied by using independent sample t-test. Linear regression analysis was used to find out whether psychological distress is a predictor of subjective well-being. One-way analysis of variance was used to find out if there are any differences among psychological distress and subjective wellbeing among different levels of hearing impairment in older adults. Ethical clearance was obtained from the ethical and research committee of the Lahore College for Women University, Lahore with IRB number 138 and issue date of 13-01-20.

\section{RESULTS}

Of the 200 participants 100 (50\%) subjects were men and $100(50 \%)$ were women. Overall mean age was $65.92 \pm 9.7$. Majority $138(69 \%)$ participants were living in nuclear family system. Most of the participants $139(69.5 \%)$ were married, $123(61.5 \%)$ belonged to middle class. Education statistics showed that $105(52.5 \%)$ participants were educated and 95(47.5\%) were uneducated. Majority 101(50\%) were having moderate hearing disability, $71(33.5 \%)$ with mild, 22(11\%) with severe and $6(3 \%)$ were dealing with profound hearing impairment.
Gender differences in psychological distress and subjective well-being in both genders were studied. Psychological distress was reported to be higher in males $(p<0.05)$ as compared to females. But no gender differences were found in subjective well-being $(p>0.05)$ (Table-I).

Linear regression analysis was used to find out the predictive relationship between psychological distress and subjective well-being in adults with hearing impairment. Results indicate that psychological distress was significant predictor of subjective well-being $(\mathrm{p}<0.01)$ (Table-II).

One-way analysis of variance was employed to test if there are any differences in perceived psychological distress and subjective well-being among different levels of hearing impairment. Results revealed that there were no significant differences found for both variables in different levels of hearing impairment $(\mathrm{p}>0.05)$ (Table-III).

\section{DISCUSSIONS}

Hearing impairment is the most common sensory disability in older adult. Review of the previous literature suggests that hearing impairment is negatively associated with the mental health and the quality of life of adults. ${ }^{19}$ In spite of importance of hearing in our life, hearing loss and especially age related hearing loss is often unrecognized and untreated issue. Even people with hearing impairment often do not consider it as a serious problem, as in many societies there is a misconception that age related hearing impairment

Table-II: Linear Regression analysis Predicting Subjective Well Being from Psychological

Distress $(n=200)$.

\begin{tabular}{lccccc}
\hline Predictor Variable & $B$ & $S E$ & $B$ & $T$ & $P$ \\
\hline Psychological & 0.17 & 0.06 & 0.18 & 2.67 & 0.00 \\
$\quad$ Distress & & & & & \\
$\mathrm{R}$ & 0.18 & & & & \\
$\mathrm{R}^{2}$ & 0.03 & & & & \\
$\mathrm{~F}$ & 7.13 & & & & \\
\hline
\end{tabular}

Note: Criterion Variable: Subjective Well-being, $\mathrm{p}<0.01$. 
Yasmeen Niazi et al.

Table-III: Summary of one-way analysis of variance (ANOVA).

\begin{tabular}{llccccc}
\hline Variables & Groups & SS & Df & $M S$ & $F$ & $P$ \\
\hline \multirow{2}{*}{ Psychological Distress } & Between Group & 136.49 & 3 & 45.49 & 2.29 & 0.07 \\
& Within Group & 3880.61 & 196 & 19.79 & & \\
& Total & 4016.55 & 196 & & & \\
Subjective Well-being & Between Group & 57.49 & 3 & 19.16 & 1.11 & 0.34 \\
& Within Group & 3381.62 & 196 & 17.25 & & \\
& Total & 3439.12 & 196 & & \\
\hline
\end{tabular}

Note: $\mathrm{SS}=$ sum of squares; $\mathrm{df}=$ degree of freedom; $\mathrm{MS}=$ mean squares. $\mathrm{p}>0.05$.

is the part of normal aging process rather than an unusual disability. As a result of it people tend to neglect the issue and do not take any serious and timely actions to seek the proper treatment which may help stop the progression of problem.

Gender differences in subjective well-being and psychological distress in adults with hearing impairment were explored in the study. No gender differences were found in case of subjective wellbeing, but for psychological distress men scored significantly higher as compared to women. In old age most of the people consider age related hearing impairment as the part of normal aging process. That is the main reason of not having a major impact of the problem on their subjective well-being. Current findings are in line with the previous literature, where hearing loss was found to be associated with psychological distress in both men and women, but slightly more depressive symptoms were observed in men than it may affect women. It may be due to the reason that men experience an additional source of psychological distress due to the fact that they usually face the societal pressure of being the bread-winner for the family, and the disability hamper their daily life functioning to a greater extent. ${ }^{20}$ Gender based association of depression and cognitive decline was studied, indicating that having both visual and hearing disability was be linked with more cognitive decline leading to higher level of depression in men, as compared to women. ${ }^{21}$ In a study aimed to investigate the association among hearing impairment and mental health in older adults, it was suggested that hearing loss was highly correlated to depressive symptoms in male population than females. ${ }^{22}$ Significantly higher association among hearing impairment and depression was observed in men at their 40s to $50 \mathrm{~s}$ as compared to those in their $60 \mathrm{~s}$. But no significant gender differences were found in both age groups in case of depression related to hearing impairment. ${ }^{23}$ The social acceptance of older adults with hearing impairment can be factor that lead to less depressive symptoms in older population as compared to young people.

Results of the study revealed that psychological distress is a predictor of subjective well-being among older adults with hearing impairment. These findings correspond with the existing literature suggesting that hearing loss actually cuts off the person facing problem form other people of society. People facing problems in communication with others may experience withdrawal from social interactions, feeling of being excluded, poor quality of life and loneliness that lead to the feeling of depression and having higher rate of psychological distress. ${ }^{12}$ People with hearing impairment are at more risk related to maladjustment in social situations, they are susceptible to experience additional disability by having difficulty in facing environmental hazardous. All these factors contribute to the decreased social health that has a significant negative influence on the mental health and well-being of individuals facing the problem. ${ }^{10}$

A study intended to explore the quality of life of people having hearing loss proved that hearing impairment was positively associated with psychological distress, where people experiencing more psychological distress tend to have decreased overall quality of life. ${ }^{18}$ Loneliness and depressive symptoms were also proved to be the significant predictors of poor physical and mental health related quality of life of people with hearing loss. ${ }^{14}$ Role of social exclusion and other mental health demographics in suicidal ideation were studied, it was evident that the psychological distress, age and severity of hearing loss were the risk factors or predictors of suicidal ideation while psychological well-being was indicated as negative predictor of suicidal ideation..$^{16}$ People suffering from hearing loss experience depression which is often linked to substantial comorbid impairment that can negatively influence the daily life responsibilities. This situation leads to the cognitive decline, anxiety and low mental well-being. ${ }^{9}$ 
Subjective wellbeing and psychological distress in older adults dealing with different levels of hearing impairment was studied. Present study provided evidence that no significant differences were found in both variables among varying levels of hearing impairment. It is evident from the literature that problem in high or medium frequency hearing has nearly no impact on mental health if low frequency hearing was not affected. ${ }^{24}$ It is well documented that if the problems related to hearing loss are understood properly and new strategies helpful for the improvement of different levels of hearing impairment are learned, this leads to improved self-perception of quality of life. Hearing loss can have negative influence on people's daily life activities, social activities and emotional state but these individuals usually do not develop the symptoms of clinical depression and anxiety if they are provided with proper social support. ${ }^{25}$ On the contrary some studies suggest that individuals with moderate and greater hearing impairment have more chances of getting the depressive symptoms as compared to those with mild or no hearing impairment. ${ }^{5}$ This lack of association may be due to some cultural differences that can influence the relationship between hearing impairment and mental health in older adults, and also due to the fewer participants in severe and profound hearing impairment groups.

Implications: This study will provide an important insight for health care practitioners to introduce new plans and policies to figure out the issue at initial level and to prevent mental health problems and improve the well-being of adult population with hearing impairment. As the age related hearing impairment is slightly unnoticeable disability, so it can be easily neglected by the people, especially if they are having any other major health related problems. This situation necessitate the formulation of effective framework for the timely identification of the problem and to ensure the availability of proper hearing aids and other assistive techniques to the people already having or at risk of hearing loss. It is recommended that professionals working with this population should carry out screening for psychological distress along with the physical monitoring of hearing loss. Social support is an important aspect regarding the management and rehabilitation of older adults with hearing impairment.
Limitations of the study: Sample of research comprised of participants from few public sector hospitals of only one city, it was mainly due to lack of resources. Psychological impact of the hearing impairment may vary in different areas or countries. To resolve the concerns related to external validity studies should include multiple public and private sector institutes form different areas of Pakistan. Future researches are needed to elucidate the relationship of hearing impairment and mental health involving more personal and environmental factors more importantly.

\section{CONCLUSION}

Gender appear to be a variant in Psychological distress reflecting high distress in men with the said condition however for the subjective well-being there were no gender differences observed. Moreover, psychological distress was found to be a predictor of subjective wellbeing in adults with hearing impairment and no significant differences in psychological distress and subjective well-being were found in different levels of hearing impairment.

Disclaimer: This article was presented at $7^{\text {th }}$ International Conference "Positive Psychology: Developing a Flourishing Community" at NIP, Quaid-e-Azam University, Islamabad, Pakistan, November 2018.

Conflict of Interest: One of the co-author of this research is member of research ethics committee.

\section{Source of Funding: None.}

Grant support and Financial Disclosures: None.

\section{REFERENCES}

1. WHO. Deafness and hearing loss. Fact sheet $N^{\circ} 300$. Mar 1 , 2020. Retrieved from: http://www.who.int/mediacentre/ factsheets/fs300/en/ [Cited 2020 Mar 16]

2. Muazzam A, Jabeen T. Development of Psycho-social Problems Scale for hearing Adults of Deaf Parents. Sci Int. 2016;28(1):695-700.

3. Hussain B, Qasim M, Masoud M, Khan L. Hearing impairments, presbycusis and the possible therapeutic interventions. Biomed Res Int. 2017;4:1228. doi: 10.15419/ bmrat.v4i4.159

4. Ejaz B, Muazzam A, Anjum A, Pollock G, Nawaz R. Measuring the Scale and Scope of Social Anxiety among Students in Pakistani Higher Education Institutions: An Alternative Social Anxiety Scale. Sustainability. 2020;12(6):2164. doi:10.3390/ su12062164 
5. Contrera KJ, Betz J, Deal JA, Choi JS, Ayonayon HN, Harris T, et al. Association of hearing impairment and emotional vitality in older adults. J Gerontol B-Psychol 2016;71(3):400-404.

6. Mehboob S, Rafi T, Ahmed N, Mehjabeen. Association of hearing loss with depression, anxiety and stress in patients suffering from Chronic Suppurative Otitis Media. Pak J Med Sci. 2019;35(2):510-514. doi:10.12669/pjms.35.2.152

7. Cheema K, Awan N, Raza N, Khan K, Qamar S, Ashraf S. Association of Comorbid Conditions with Sixmonth Survival and Disease Outcome in Patients of Necroinflammatory Otitis Externa. J Coll Physicians Surg Pak. 2020;30:498-502. doi: 10.29271/jcpsp.2020.05.498.

8. Muazzam A, Ahmad A. Predictors of sleep disturbances among college students: Interplay of media exposure and Health related quality of Life. Pak J Psychol Res. 2017;32(2):393-406.

9. Cosh S, Helmer C, Delcourt C, Robins T, Tully P. Depression in elderly patients with hearing loss: Current perspectives. Clin Interv Aging. 2019;14:1471-1480. doi: 10.2147/CIA.S195824

10. Mumtaz N, Saqulain G.Hospital and health administrator level barriers and priorities for National Neonatal Hearing Screening in Pakistan: A thematic analysis. Pak J Med Sci. 2020;36(5):1036-1041. doi: 10.12669/pjms.36.5.1965

11. Ilyas Z, Muazzam A. Development and validation of a general adjustment to aging scale in Pakistan. J Art Soc Sci. 2015;2(2):81-96

12. Iwagami M, Kobayashi $\mathrm{Y}$, Tsukazaki E, Watanabe $\mathrm{T}$, Sugiyama T, Wada T, et al. Associations between selfreported hearing loss and outdoor activity limitations, psychological distress and self-reported memory loss among older people: Analysis of the 2016 Comprehensive Survey of Living Conditions in Japan: Hearing loss and health outcomes. Geriatr Gerontol Int. 2019;19(18):747-754. doi:10.1111/ggi.13708

13. Diener E. Subjective well-being: The science of happiness and a proposal for a national index. Am Psychol. 2000;55(1):34-43. doi:10.1037/0003-066X.55.1.34

14. Dean G, Orford A, Staines R, McGee A, Smith K. Psychosocial well-being and health-related quality of life in a UK population with Usher syndrome. BMJ Open. 2017;7(1). doi: 10.1136/ bmjopen-2016-013261

15. Heffernan E, Coulson N, Henshaw H, Barry J, Ferguson M. Understanding the psychosocial experiences of adults with mild-moderate hearing loss: An application of Leventhal's self-regulatory model. Int J Audiol. 2016;55. doi:10.3109/1 4992027.2015.1117663

16. Akram B, Nawaz J, Rafi Z, Akram A. Social exclusion, mental health and suicidal ideation among adults with hearing loss: Protective and risk factors. J Pak Med Assoc. 2018;68:388-393.
17. Furukawa TA, Kessler RC, Slade T, Andrews G. The performance of the $\mathrm{K} 6$ and $\mathrm{K} 10$ screening scales for psychological distress in the Australian National Survey of Mental Health and Well-Being. Psychol Med. 2003;33(2):357-62. doi: 10.1017/S0033291702006700

18. Pavot W, Diener E. Review of the Satisfaction with Life Scale. Psychol Assess. 2009;5(2):164-172. doi:10.1007/97890-481-2354-4 5

19. Nordvik O, Heggdal P, Brannstrom J, Vassbotn F, Aarstad AK, Aarstad HJ. Generic quality of life in persons with hearing loss: A systematic literature review. BMC Ear Nose Throat Disord. 2018;18(1). doi:10.1186/s12901-018-0051-6

20. Shamim A, Muazzam A. Positive Emotions and Compliance as Predictors in the Management of Type II Diabetes. Pak J Soc Clin Psychol. 2018;16(2):27-33.

21. Lyu J, Kim HY. Gender-Specific Associations of Sensory Impairments with Depression and Cognitive Impairment in Later Life. Psychiatry Investig. 2018;15(10):926-934. doi: $10.30773 /$ pi.2018.06.28.2

22. Shin HY, Hwang HJ. Mental Health of the People with Hearing Impairment in Korea: A Population-Based Cross-Sectional Study. Korean J Fam Med. 2017;38:57-63. doi: 10.4082/ kjfm.2017.38.2.57

23. Keidser G, Seeto M. The Influence of Social Interaction and Physical Health on the Association Between Hearing and Depression with Age and Gender. Trends Hear. 2017;21:115. doi: $10.1177 / 2331216517706395$

24. Tambs K. Moderate Effects of Hearing Loss on Mental Health and Subjective Well-Being: Results from the Nord-Trondelag Hearing Loss Study. Psychosom Med. 2004;66:776-782. doi: 10.1097/01.psy.0000133328.03596.fb

25. Chen A, Most T. Auditory, Visual, and Auditory-Visual Perception of Emotions by Individuals with Cochlear Implants, Hearing Aids, and Normal Hearing. J Deaf Stud Deaf Educ. 2009;14(4): 449-464.

\section{Authors' Contribution:}

YN: Conceived, designed and did statistical analysis \& editing of manuscript. She is also responsible and accountable for the accuracy or integrity of the work.

BE: Supervised research.

AM: Did review and final approval of manuscript. 